\title{
Antigen and Memory CD8 T Cells: Were They Both Right?
}

\author{
Slava Epelman, MD, PhD and Christopher H. Mody, MD
}

\begin{abstract}
Picture yourself as a researcher in immunology. To begin your project, you ask a question: Do CD8 $\mathrm{T}$ cells require antigen to maintain a memory response? This question is of prime importance to numerous medical fields. In chronologic order, you digest the literature, but unfortunately, you hit a major stumbling block in the 1990s. The crux of the problem is that which so often happens in science: two well-recognized, capable groups emerge with diametrically opposed conclusions, leaving you pondering which set of wellcontrolled data to believe. Fortunately, years later, a surprising group of articles sheds light on this mystery and subtly reconciles these two positions.
\end{abstract}

Key words: antigen, CD8 cells, central memory, effector memory

C ytotoxic CD8 T cells are important mediators of host defense, and their role in protective immunity has recently been highlighted by concerns of possible widespread pandemic viral infections. Designing strategies to immunize and therefore protect vulnerable populations requires a thorough understanding of the complex factors involved in the generation of CD8 memory cells. However, there has been considerable debate over the years about the nature of CD8 memory cells, whether they truly exist, and if so, what signals, if any, keep them alive for such an extended period of time. Memory cells are small and long-lasting, proliferating slowly to maintain the size of the memory pool. ${ }^{1,2}$ If memory cells were quiescent, there would be no need to regulate them. Understanding the mechanisms involved in the propagation of CD8 T-cell memory is critical to numerous areas of research, including virus-host interactions, vaccine development, autoimmune diseases, and cancer immunology. With that, the stage is set for the retelling of an interesting scientific debate that had its beginning in the early 1990s and its end in recent years. The debate was based on the following question: Do memory CD8 T cells require the presence of antigen $(\mathrm{Ag})$ to survive?

Slava Epelman: Department of Microbiology and Infectious Diseases, University of Calgary, Calgary, AB; currently Department of Internal Medicine, Cleveland Clinic, Cleveland, OH; Christopher H. Mody: Departments of Microbiology and Infectious Diseases and Internal Medicine, University of Calgary, Calgary, $A B$.

Correspondence to: Dr. Christopher H. Mody, Room 273, Heritage Medical Research Building, 3330 Hospital Drive NW, University of Calgary, Calgary, AB T2N 4N1; e-mail: cmody@ucalgary.ca.

DOI 10.2310/7480.2007.00001
Investigations into memory CD8 cells resulted in the emergence of two opposing theories, based in part on subtly different experimental approaches. Lau and colleagues and Mullbacher and Flynn argued that $\mathrm{Ag}$ was not required for efficient CD8 memory (anti-Ag), ${ }^{3,4}$ whereas Gray and Matzinger and Kundig and colleagues argued the opposite-Ag was required for CD8 memory (pro-Ag). ${ }^{5,6}$ Experimentally, both groups gave an initial intraperitoneal immunization with virus, isolated and then transferred memory CD8 cells into naive recipient mice. The anti-Ag group then rechallenged by the intravenous route (centrally), whereas the pro-Ag group rechallenged intracerebrally or in the hind footpad (peripherally). This is a critical difference, as we will come to understand. Both groups agreed that transferred memory CD8 cells persisted in the absence of $\mathrm{Ag}^{3-6}{ }^{3-6}$ However, the function of these cells during secondary challenge with virus led to divergent conclusions. The anti-Ag group launched the first salvo and demonstrated that on intravenous challenge, virusspecific CD8 cells survived for extended periods. ${ }^{3,4}$ By contrast, when the pro-Ag group challenged peripherally with virus, transferred memory cells provided initial in vivo protection, but in the absence of $\mathrm{Ag}$, protection was lost over time. ${ }^{5,6}$ The pro-Ag group countered that to have functional CD8 memory cells, as defined by in vivo protection, Ag must be present.

Why was there a discrepancy between the two groups? The experimental approaches taken by these groups capitalized on different immunologic concepts that were not known at that time but can account for the different results. Three issues explain how these divergent conclusions arose. 
To begin, although both groups gave an initial intraperitoneal immunization with virus, they employed different sites of secondary immunization. The anti-Ag group challenged by the intravenous route, which would be filtered by the spleen, whereas the pro-Ag group challenged intracerebrally or in the hind footpad, which are peripheral sites that require extravasation. A peripheral challenge would test the ability of the memory cells to move from blood to the tissue, which was a classic definition of memory cells at that time. By contrast, intravenous viral challenge, which was employed by the anti-Ag group, was testing the ability of $\mathrm{T}$ cells in the spleen to respond. It has been demonstrated that simply possessing large numbers of naive $\mathrm{T}$ cells at the site of viral challenge is sufficient for protection. For example, if unprimed mice with a transgenic T-cell receptor against a lymphocytic choriomeningitis virus (LCMV) peptide are infected with LCMV in the brain or in the hind footpad, they die, despite the presence of large numbers of Ag-specific CD8 cells. However, if you give LCMV by the intravenous route, these mice clear the infection, presumably owing to the high number of Ag-specific CD8 cells found in the spleen compared with the low number in the periphery. ${ }^{6}$

Second, the anti-Ag group used irradiated recipients for adoptive transfer, whereas the pro-Ag group did not. The pro-Ag group argued that adoptive transfer of CD8 $\mathrm{T}$ cells into an irradiated host would result in a large homeostatic expansion, resulting in an artificially inflated CD8 memory cell pool. ${ }^{7}$ The net effect would be to greatly enhance the number of Ag-specific CD8 T cells present and perhaps change their activation state (via proliferation and cytokine production) to that of $\mathrm{T}$ cells that have encountered Ag. Although the number of Agspecific CD8 $\mathrm{T}$ cells was likely to have expanded by homeostatic proliferation, homeostatic proliferation does not result in a change in the activation state of memory $\mathrm{T}$ cells. ${ }^{8}$

Lastly, both groups erred by not fully understanding the complexity of the system, and it is with a number of recent reports that clarity emerges. Two CD8 cell memory subsets have been defined, each possessing different functions and homing potential. ${ }^{9,10}$ Central memory CD8 cells $\left(\mathrm{CD} 8 \mathrm{~T}_{\mathrm{CM}}\right)$ circulate through lymphoid tissues, such as the spleen, and possess the ability to proliferate rapidly when stimulated but cannot rapidly express cytotoxic T lymphocyte (CTL) activity. Effector memory CD8 cells $\left(\mathrm{CD} 8 \mathrm{~T}_{\mathrm{EF}}\right)$ reside in or circulate through peripheral tissues, such as the lung and skin, and have rapid and potent ex vivo CTL activity. Given these new observations, the results of the pro-Ag and anti-Ag groups can now be reconciled.
The pro-Ag group adoptively transferred splenocytes, a population that was enriched for $\mathrm{CD} 8 \mathrm{~T}_{\mathrm{CM}}$ cells, a population that does not rapidly up-regulate effector functions and does not home to the periphery. ${ }^{5,6,9,10}$ When the pro-Ag group challenged mice peripherally, there were no CD8 $\mathrm{T}_{\mathrm{EF}}$ cells present, and as a result, they concluded that no memory cells were present. There were, however, CD8 $\mathrm{T}_{\mathrm{CM}}$ cells present, although they were located in the secondary lymphoid tissue. By contrast, the anti-Ag group transferred splenocytes that were allowed to expand in irradiated recipients. Homeostatic proliferation resulted in a massive number of $\mathrm{CD} 8 \mathrm{~T}_{\mathrm{CM}}$ cells that homed to the spleen and lymph nodes. Given that large numbers of cells are protective, it was not surprising to find that these animals were protected from subsequent intravenous challenge, which would direct the virus to the central compartment. Both groups transferred CD8 $\mathrm{T}_{\mathrm{CM}}$ cells, and for this reason they agreed that CD8 memory cells survive indefinitely. However, neither group analyzed the population of memory cells that provides rapid effector functions attributed to memory cells. For this, they would have had to purify CD8 memory cells from peripheral tissue to isolate CD8 $\mathrm{T}_{\mathrm{EF}}$ cells.

The identification of central and effector memory Tcell subsets finally lays to rest an interesting historical debate that raged in the literature without reconciliation and provides a unique example of how minor differences in methodology can result in dramatically different conclusions. Most groups believe that although a complex set of signals is required to maintain CD8 memory cells, Ag is not, and only brief exposure to $\mathrm{Ag}$ is sufficient to maintain T-cell memory. ${ }^{11}$ The debate is now focused on what factors influence the differentiation of CD8 memory cells into $\mathrm{T}_{\mathrm{EF}}$ or $\mathrm{T}_{\mathrm{CM}}$. Understanding how factors such as original burst size, Ag, and cytokine milieu are critical to memory cell subset differentiation will aid in understanding disease pathogenesis, vaccine development, and development of immunotherapeutics. ${ }^{11-13}$ Therefore, in the battle between the pro-Ag and anti-Ag camps, the final result was in a way a draw: both were right, although for the wrong reasons.

\section{References}

1. Selin LK, Vergilis K, Welsh RM, et al. Reduction of otherwise remarkably stable virus-specific cytotoxic $\mathrm{T}$ lymphocyte memory by heterologous viral infections. J Exp Med 1996;183:248999.

2. Marrack P, Bender J, Hildeman J, et al. Homeostasis of alpha beta TCR+ T cells. Nat Immunol 2000;1:107-11. 
3. Lau LL, Jamieson BD, Somasundaram T, et al. Cytotoxic T-cell memory without antigen. Nature 1994;369:648-52.

4. Mullbacher A, Flynn K. Aspects of cytotoxic T cell memory. Immunol Rev 1996;150:113-27.

5. Gray D, Matzinger P. T cell memory is short-lived in the absence of antigen. J Exp Med 1991;174:969-74.

6. Kundig TM, Bachmann MF, Oehen S, et al. On the role of antigen in maintaining cytotoxic T-cell memory. Proc Natl Acad Sci USA 1996;93:9716-23.

7. Kundig TM, Bachmann MF, Ohashi PS, et al. On T cell memory: arguments for antigen dependence. Immunol Rev 1996;150:63-90.

8. Murali-Krishna K, Lau LL, Sambhara S, et al. Persistence of memory CD8 T cells in MHC class I-deficient mice. Science 1999; 286:1377-81.
9. Northrop JK, Shen H. CD8+ T-cell memory: only the good ones last. Curr Opin Immunol 2004;16:451-5.

10. Masopust D, Vezys V, Marzo AL, et al. Preferential localization of effector memory cells in nonlymphoid tissue. Science 2001;291: 2413-17.

11. Haring JS, Badovinac VP, Harty JT. Inflaming the CD8+ T cell response. Immunity 2006;25:19-29.

12. Bachmann MF, Wolint P, Schwarz K, et al. Functional properties and lineage relationship of CD8+ $\mathrm{T}$ cell subsets identified by expression of IL-7 receptor alpha and CD62L. J Immunol 2005; 175:4686-96.

13. Marzo AL, Klonowski KD, Le Bon A, et al. Initial T cell frequency dictates memory CD8+ $\mathrm{T}$ cell lineage commitment. Nat Immunol 2005;6:793-9. 